\title{
AVALIAÇÃO DAS POTENCIALIDADES DA INSERÇÃO DA REALIDADE AUMENTADA EM CANTEIRO DE OBRAS
}

\author{
Fernando H. L. Silva ${ }^{(1)}$, Josyanne P. Giesta ${ }^{(2)}$, Rayanne R. D. Câmara ${ }^{(1)}$ \\ (1) Universidade Federal do Rio Grande do Norte \\ (2) Instituto Federal de Educação, Ciência e Tecnologia do Rio Grande do Norte
}

\begin{abstract}
Resumo
Com o avanço tecnológico as ferramentas Building Information Modeling (BIM) apresentamse cada vez mais capazes de atender as necessidades da indústria da Arquitetura, Engenharia e Construção (AEC). A Realidade Aumentada (RA), por exemplo, proporciona uma visão imersiva do futuro ambiente, permitindo uma integração entre o meio virtual e o real. Nesse contexto, o presente trabalho tem como objetivo avaliar a inserção da RA em canteiro de obras, buscando compreender suas potencialidades no processo de compreensão dos projetos. $\mathrm{O}$ método adotado foi o estudo de caso, partindo de observações de fenômenos reais. Assim, as principais etapas desenvolvidas foram: a) seleção de duas obras localizadas na região metropolitana de Natal/RN; b) seleção das ferramentas de RA - Augment e Augin; c) elaboração do questionário; d) teste piloto; e) coleta e análise dos dados. Como resultado, observou-se um grande potencial de contribuição da RA no meio construtivo, bem como boa aceitação por parte dos trabalhadores. Entretanto, percebeu-se como principal dificuldade a necessidade de internet com boa capacidade. Diante do cenário apresentado, entende-se que esse processo se dará de forma lenta e gradual, visto que a implementação de RA requer investimentos para aquisição de equipamentos, treinamentos e licenças de softwares.
\end{abstract}

\section{Introdução}

O crescimento acelerado das tecnologias computacionais vem impactando de forma positiva no desenvolvimento de projetos e execução de obras de pequeno e grande porte. A inclusão dos processos, políticas e tecnologias agregadas ao Building Information Modeling (BIM) elevam o nível de precisão do mercado da construção civil. O BIM permite aos projetistas uma visão mais real do projeto em pleno desenvolvimento, ocasionando reduções de erros e interferências que geralmente só são identificados durante ou somente após a execução, acarretando em problemas que podem gerar custo adicional no valor final da obra.

Os ganhos com a inclusão dos modelos virtuais estão além da fase de elaboração, visto que visualizações mais próximas da realidade propiciam uma melhor compreensão dos projetos [1]. 
Mediante esse processo de renovação, a Realidade Aumentada (RA) pode ser utilizada para melhor entendimento e compreensão sobre o meio, principalmente no que diz respeito ao ambiente construído [2]. A RA permite que o usuário se mantenha em seu ambiente físico e o ambiente virtual seja inserido dentro do espaço do usuário, possibilitando a visualização do projeto virtual.

Haja vista os ganhos gerados com combinação do BIM e a RA, empresas estão sendo induzidas a elevar seus níveis de investimentos em relação a tecnologia para otimizarem seus fluxos de trabalhos, permitindo promover a informação e o acompanhamento de seus processos [3]. Nesse sentido, a busca pelas novas tecnologias no intuito de aumentar a produtividade e a qualidade de seus entregáveis vem aumentando de forma acelerada.

Entre as vantagens de utilizar o BIM e a RA no canteiro de obras estão a possibilidade de alimentar informações no escritório ou diretamente na obra, de maneira que os arquivos atualizados sejam acedidos em um curto espaço de tempo por qualquer envolvido no processo; a compatibilização e acompanhamento entre o que é projetado e planejado com o que foi efetivamente executado; e até evitar problemas por falta de comunicação entre as equipes de projeto e execução [4].

Os erros de projetos que são remetidos a decisões no canteiro de obras, podem apresentar como consequências: perda de produtividade, comprometimento do que está sendo executado e a falta de conformidade da obra em relação ao projeto [5].

No caso deste estudo, busca-se enfatizar a relevância da inclusão da RA no dia a dia do canteiro de obras, para que se possa ter um entendimento mais próximo da realidade de como ficará o futuro empreendimento, resultando assim na redução de erros e retrabalho e consequentemente na perda de tempo e de recursos financeiros.

Dito isto, este artigo tem como objetivo avaliar a inserção da RA em canteiro de obras, buscando compreender suas potencialidades no processo de compreensão dos projetos.

\section{Referencial teórico}

Com o acelerado avanço da tecnologia e o surgimento de novos aplicativos desenvolvidos especificamente para dispositivos móveis com objetivo comercial nos campos da Arquitetura, Engenharia e Construção (AEC), estudos voltados para essa realidade passam a ser praticados. Por apresentar grande capacidade e a simplificação de hardware, é possível notar um aumento na aplicação da RA [6]. Por exemplo, [1] em seu estudo aplicaram a RA para utilização na visualização de projetos de instalações para a execução no canteiro de obras e concluíram que a exploração das novas formas de visualização dos modelos tridimensionais no canteiro de obras agrega valor. Já [7] estudaram as possibilidades do uso da RA em dispositivos móveis para AEC, onde relataram em sua pesquisa algumas dificuldades iniciais na aplicação dos dispositivos e concluíram que a RA é uma tecnologia que desperta interesse para o setor AEC e pode ser utilizada como uma ferramenta adicional na busca pela melhoria dos processos.

Já [8] criaram um protótipo de um sistema de RA que sobrepõe modelos virtuais 3D e permite que usuários concedam feedback a respeito dos projetos, assim, permitindo a ferramenta auxiliar a participação pública no planejamento urbano.

E [9] propuseram em seu estudo a aplicação da RA para avaliar a capacidade do BIM de criar ambientes realistas a fim de desenvolver a colaboração, comunicação e aprendizado. 
Bem como, [10] apontam os principais usos, que são: simulação e visualização, comunicação e colaboração e acesso à informação ou avaliação; os autores identificaram inúmeros benefícios atrelados a sua aplicação na construção civil, destacando-se a comparação entre o projetado e o construído.

[2] publicaram um estudo intitulado: validação de aplicativo comercial visando à incorporação da realidade aumentada a um modelo de avaliação pós-ocupação, onde as pesquisadoras apontam deficiências que ainda inviabilizam a validação da solução tecnológica.

Em todos esses casos, [7] relatam que a preocupação comum é o posicionamento preciso, a escalabilidade e conexão de dados. Onde o posicionamento depende do método de registo adotado. [11] mencionam em seu estudo que existem três métodos para que o modelo possa ser posicionado: a) com base em marcador, onde se utiliza tecnologias de rastreamento padrão para sobrepor os objetos virtuais; b) GPS, giroscópio, acelerômetros e bússola; c) pontos específicos na imagem, que utiliza tecnologias sem marcadores.

\subsection{Aplicativos de RA que podem ser utilizados na indústria da construção}

Apesar de ainda possuir uso restrito na indústria AEC, mas que vem crescendo de forma progressiva, os aplicativos de RA para dispositivos móveis dispõem de uma grande variedade no meio comercial, que engloba desde produtos pagos com versões de avaliação até os com assinaturas totalmente gratuitas.

O Augin tem origem brasileira e trabalha com a tecnologia RA para construção com fluxos automáticos, por meio de plug-in, para o envio de arquivo de imagem em $3 \mathrm{D}$, que permite ao usuário fazer usos de todas as suas funcionalidades disponíveis sem nenhum custo. Por outro lado, o Augment, criado em 2011, por uma startup francesa com o intuito de trabalhar com uma plataforma para visualização de produtos em 3D e RA para acelerar os processos de aprovação de projetos e redução de custos de prototipagem fornece uma avaliação gratuita de 14 dias.

A Tabela 1 apresenta uma descrição dos aplicativos Augin e Augment para uso em AEC.

Tabela 1: Comparativo de propriedades

\begin{tabular}{lcc}
\hline Propriedade & \multicolumn{2}{c}{ Aplicativos } \\
\hline Fabricante & Augin & Augment \\
Sistema operacional & Android/IOS & Android/IOS \\
Versão Android & 3.10 & 4.0 .8 -beta+30650 \\
Entrada de dados & Interface gráfica com usuário & Interface gráfica com usuário \\
Permite inserir objetos 3D & Integração: 3DS Max \\
Custo & Gratuito & Sim \\
\hline
\end{tabular}

\subsection{Aplicativos de Realidade Mista}

A Realidade Mista (RM) é uma ferramenta de grande potencial para o uso na AEC. Seu conceito que envolve a Realidade Virtual (RV) e RA, permite mesclar o meio real com o meio virtual possibilitando a interação entre eles. O Hololens da Microsoft, por exemplo, permite que [12] toque, segure e mova hologramas naturalmente de forma muito semelhante a objetos reais. Outra ferramenta que faz uso dessa tecnologia é o Unity Reflect, que possibilita [13] trazer 
diversos modelos BIM com todos os metadados para 3D em tempo real, mantendo um vínculo ativo entre eles, admitindo ainda transferência de modelos nativos do Revit, Navisworks, SketchUp e Rhino.

\section{Métodos e técnicas}

O método aplicado foi o estudo de caso, que segundo [14] consiste em um estudo profundo e exaustivo de um ou poucos objetivos, de maneira que permita seu amplo e detalhado conhecimento. No caso específico, partiu-se de observações de fenômenos reais com o auxílio de smartphones e tablets. Deste modo, as principais etapas desenvolvidas foram (Figura 1): a) delineamento da pesquisa; b) desenho da pesquisa; c) preparação da coleta de dados; d) coleta de dados; e e) análise dos dados e conclusão.

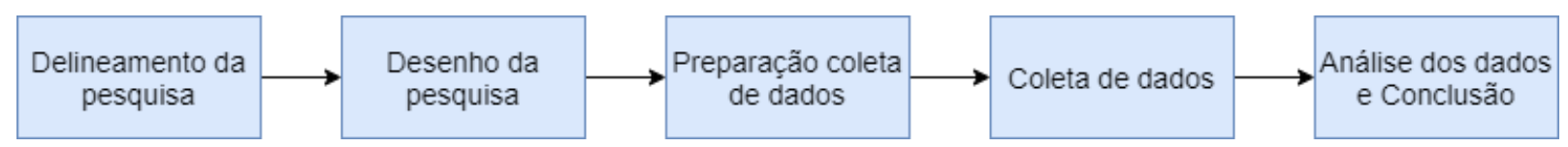

Figura 1: Etapas do estudo de caso.

A etapa de delineamento da pesquisa teve como base a realização da revisão bibliográfica na temática, essa pesquisa se deu sem sistematização rigorosa nas buscas. Na etapa de desenho da pesquisa fez-se a definição dos dois estudos de caso e a elaboração do protocolo que orientou a etapa de coleta de dados. Na terceira etapa, de preparação das coletas de dados, entrou-se em contato com os casos selecionados, as duas obras e agendou-se as datas das entrevistas. Ainda nessa etapa, foi realizado o teste piloto. Após a aplicação dos questionários, que correspondeu a etapa de coleta de dados, iniciou-se a análise dos dados e conclusão.

Para a aplicação dos questionários, foi necessário o desenvolvimento de projetos referentes as obras em questão, para tanto utilizou-se o software Autodesk Revit (2019), sendo o modelo exportado para as ferramentas de RA através de plugins específicos indicado pelos fabricantes. O plugin OBJ Export 2 extrai o modelo 3D em arquivo object 3D, além de exportar texturas no formato png. Esse grupo de arquivos é compactado em formato zip e inserido na plataforma online do Augment que, por sua vez, gera o modelo em RA.

Já o Augin disponibiliza o plugin Auge que exporta o arquivo em formato RVT para a plataforma do aplicativo. Porém essa versão não exporta texturas. Atualmente a versão 3.10 do Augin permite coordenar em um mesmo modelo 3D, diferentes disciplinas BIM, como arquitetura, estrutura e complementares, para posteriormente filtrá-las em Realidade Aumentada, ou seja, cria um modelo federado ou integrado a partir do arquivo IFC ou FBX.

As ferramentas de RA utilizadas nessa pesquisa foram selecionadas com base no conhecimento da equipe de treinamento, bem como aplicabilidades e facilidades de acesso. O propósito foi mostrar as potencialidades dos aplicativos e também suas divergências, como por exemplo, a necessidade de utilizar o Alvo Padrão do Augin em aparelhos celulares ou tablet, que não são capazes de rodar as plataformas; devido aparelhos com sistemas androides e IOS sem a tecnologia AR Core/AR Kit necessitarem de um alvo padrão fornecido pelo fabricante para posicionar os modelos. A versão atual do Augin conta com um documento para referenciar o projeto e aumentar a precisão do posicionamento do modelo de RA no ambiente real, denominado de Reference Tracker. 
O questionário, composto por 21 perguntas (objetivas e subjetivas), foi elaborado com o intuito de coletar as informações das experiências vividas por cada participante do treinamento durante o uso das ferramentas de RA no canteiro de obras. A estrutura foi dividida em quatro etapas (Figura 2): a) caracterização da obra; b) perfil do profissional entrevistado; c) introdução ao Building Information Modeling; e d) Realidade Aumentada.

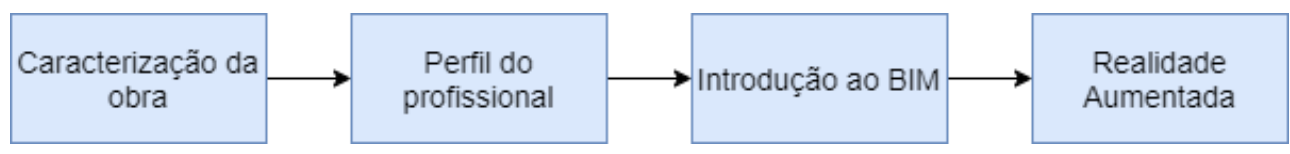

Figura 2: Estrutura do questionário.

O teste piloto iniciou-se com a capacitação prévia da equipe de execução, introduzindo os conceitos a respeito do BIM, apresentando alguns casos de usos e as principais vantagens da utilização desta metodologia, como a facilidade de acesso às informações do projeto, extração de quantitativos, visualização do modelo 3D, entre outras. Por fim, foram explanados os conceitos de Realidade Aumentada e a sua aplicabilidade no canteiro de obras, apresentando os modelos dos projetos que estão sendo executados.

\section{Estudo de caso}

O estudo de caso se deu pela coleta e análise de dados através de um questionário elaborado e aplicado no decorrer das visitas e reuniões não estruturadas realizadas. Ao todo o questionário foi aplicado com 24 colaboradores distribuídos em 2 obras; residências unifamiliares do tipo duplex, localizadas em condomínio fechado na região metropolitana de Natal/RN.

Inicialmente, realizou-se um treinamento com os engenheiros e mestres de obra (Figura 3), que posteriormente repassaram os conhecimentos para os demais colaboradores, para que, assim, pudessem obter o melhor aproveitamento durante a utilização in loco.

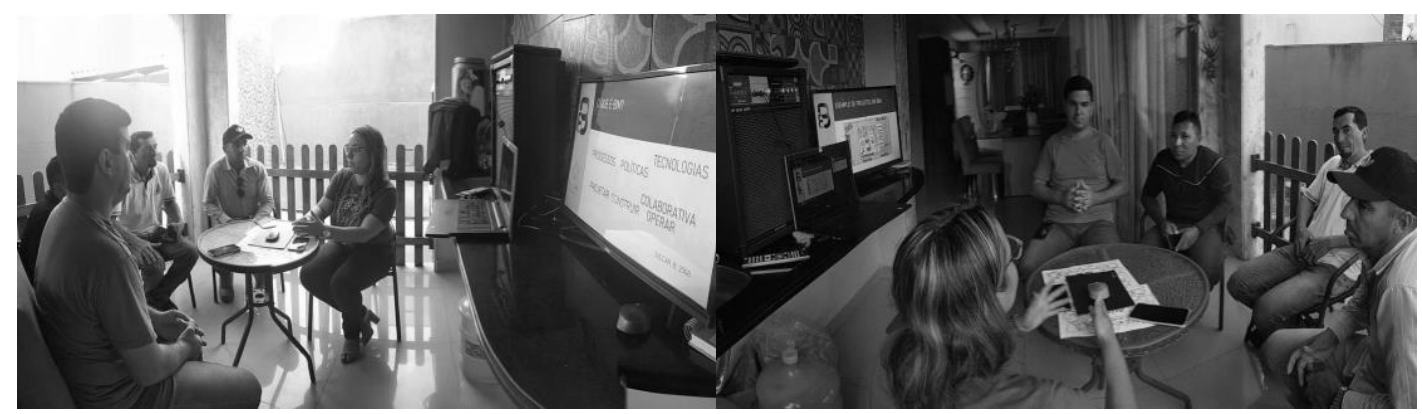

Figura 3: Treinamento inicial - introdução ao BIM e RA.

Em seguida, prosseguiu-se para a primeira obra, onde foi aplicado, in loco, juntamente com os demais colaboradores, as ferramentas propostas. Ao término da experiencia, foi aplicado o questionário de forma online para que os participantes pudessem responder todas as questões apresentadas. Ao término da aplicação na primeira obra, os envolvidos se dirigiram para a segunda, onde realizaram os mesmos procedimentos acima descritos. Após toda aplicação, os dados obtidos foram compilados e analisados. 
Ainda, neste experimento, quanto aos modelos, os mesmos foram posicionados de forma aleatória em ambos os apps, pois a versão utilizada não tinha nenhum tipo de referenciamento in loco.

\section{Resultados e discussões}

As imagens representadas abaixo referem-se a uma das obras em que foi aplicada o uso da RA, onde a Figura 4a representa a modelagem desenvolvida na ferramenta Autodesk Revit 2019, seguida do modelo exportado pelo aplicativo Augin (Figura 4b), aplicação do Augment in loco (Figura 4c) e por último (Figura 4d) o empreendimento executado. Nota-se a ausência de texturas no modelo exportado pelo Augin (Figura 4b), pois este não lê texturas do arquivo RVT, enquanto no Augment consegue-se visualizar algumas.
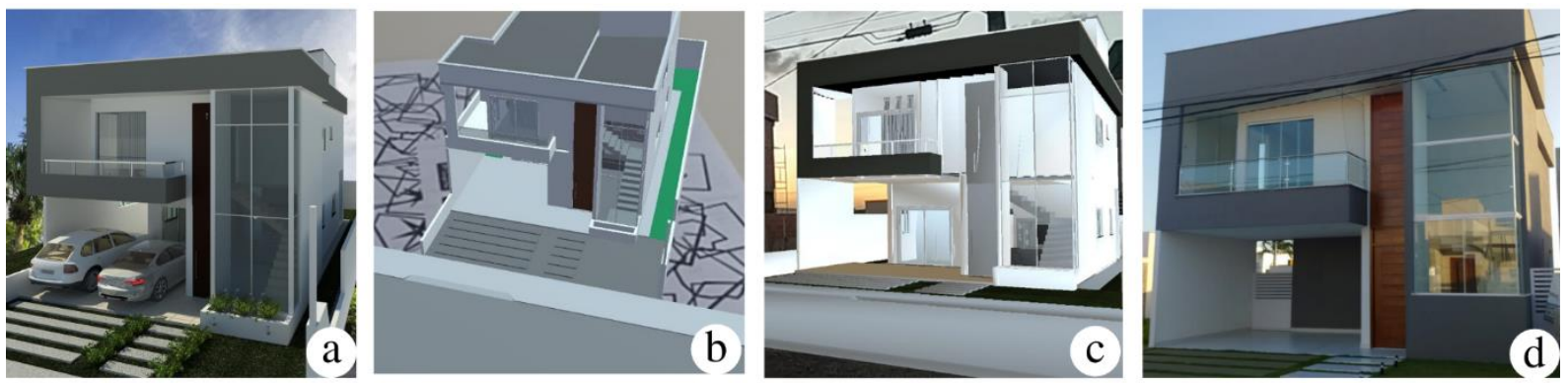

Figura 4: Comparativo de ferramentas.

No que se refere aos resultados encontrados, a partir da análise dos questionários e reuniões não estruturadas, as respostas foram examinadas e estão explanadas a seguir.

\subsection{Faixa etária dos profissionais}

De acordo com o gráfico da Figura 5, os empreendimentos apresentavam profissionais com a faixa etária variando entre 18 e 45 anos de idade, onde nas faixas de 18 a 25 anos e 31 a 35 anos apresentaram os menores índices.

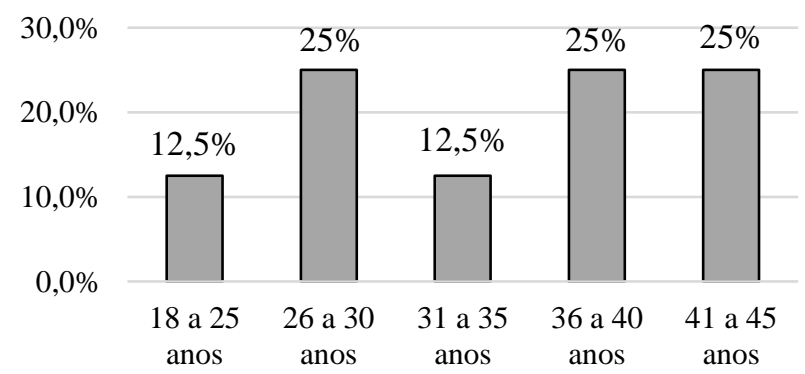

Figura 5: Faixa etária dos profissionais.

\subsection{Funções desempenhadas pelos profissionais}

Conforme o gráfico apresentado na Figura 6, a função de servente e pedreiro são responsáveis por mais de $65 \%$ de todo o percentual apresentado. As demais funções não apresentaram 
diferenças significativas em seus percentuais. Vale ressaltar, que o questionário foi aplicado apenas para aqueles que estavam presente no dia da aplicação.

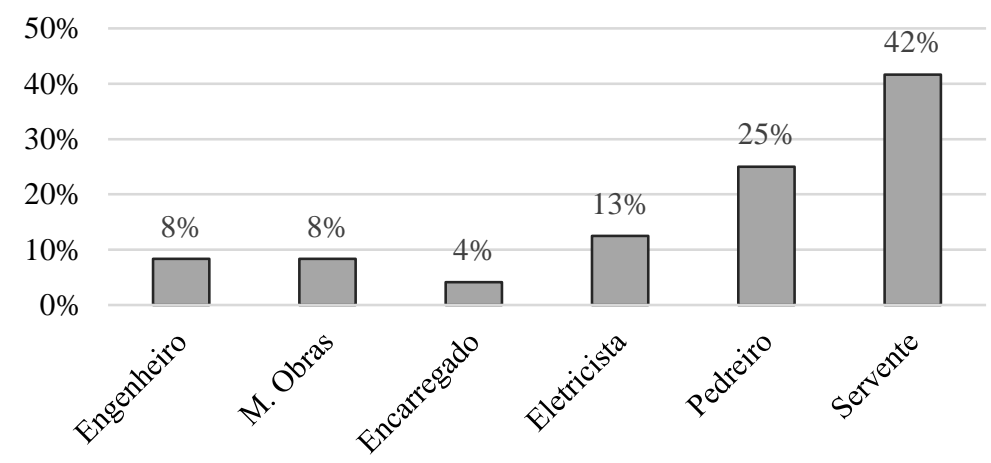

Figura 6: Função dos profissionais.

\subsection{Quanto a utilização da Realidade Aumentada no canteiro de obras}

No que tange ao uso da RA no canteiro, podemos ver no gráfico da Figura 7 que a grande maioria nunca utilizou a RA na execução das obras em que participaram. Tal fator, pode se dar pela falta de conhecimento das ferramentas; outro fator que também pode ser associado a este cenário é que nas demais obras em que participaram, os projetos não foram realizados com a aplicação da metodologia BIM, dificultando ou até impossibilitando a utilização dessas ferramentas no decorrer da execução do projeto.

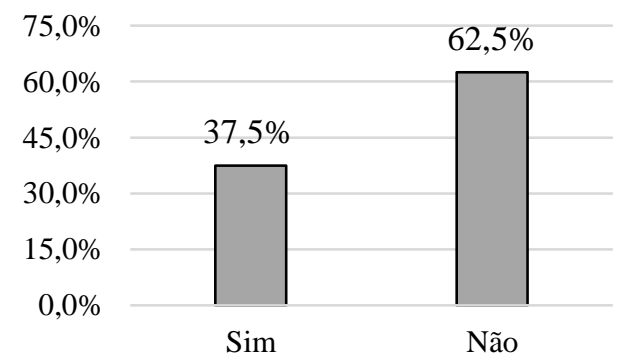

Figura 7: Já utilizou a RA no canteiro de obras.

\subsection{Quanto a facilidade de aplicação do app no canteiro de obras}

O gráfico da Figura 8 apresenta a preferência dos usuários após a utilização do Augin e do Augment. A maioria dos colaboradores deu preferência ao Augment, tendo em vista que em seus relatos a maioria mencionou a dificuldade que tiveram em posicionar o modelo no Augin, haja vista que nem todos os dispositivos móveis dispõe de tecnologia voltada a Realidade Aumentada, sendo necessário a utilização de um alvo padrão para proceder com o modelo; houveram casos em que o Augin não foi suportado no dispositivo móvel.

O Augment ficou limitado quanto a sua imersividade dentro do modelo, tendo em conta que o mesmo não permite sua navegação dentro dos ambientes internos, diferentemente do Augin, que permite imersividade total do modelo.

Em ambos os apps foram constatados dificuldades, tais como: (a) necessidades de usar a internet durante a utilização; (b) dificuldades de posicionamento do modelo no local; (c) 
dificuldade em ajustar a escala; (d) perda de elementos, texturas e redução dos níveis de detalhamento apresentados na modelagem original.

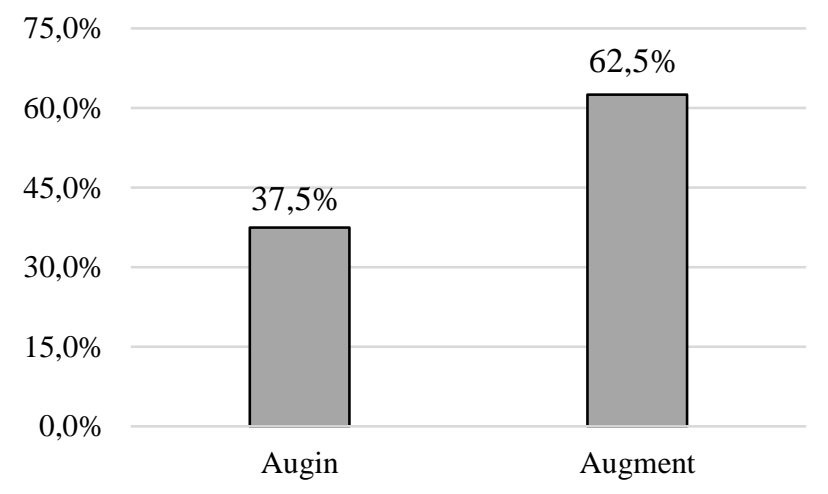

Figura 8: Quanto a facilidade de aplicação do app.

\subsection{Opinião quanto a aplicação da Realidade Aumentada no canteiro de obras}

A maioria dos participantes que utilizaram as ferramentas e que tiveram pela primeira vez o contato com a Realidade Aumentada demonstraram boa aceitação e destacaram que é possível a sua utilização no dia a dia da obra (Figura 9), facilitando assim a compreensão dos projetos que as vezes são mal detalhados e visualizando informações necessárias que se perdem em planta. Alguns relataram ainda, a dificuldade de receber um projeto que não apresente falhas, onde boa parte só é percebida no decorrer da execução, acarretando transtornos que dificultam o desenvolvimento da execução do empreendimento.

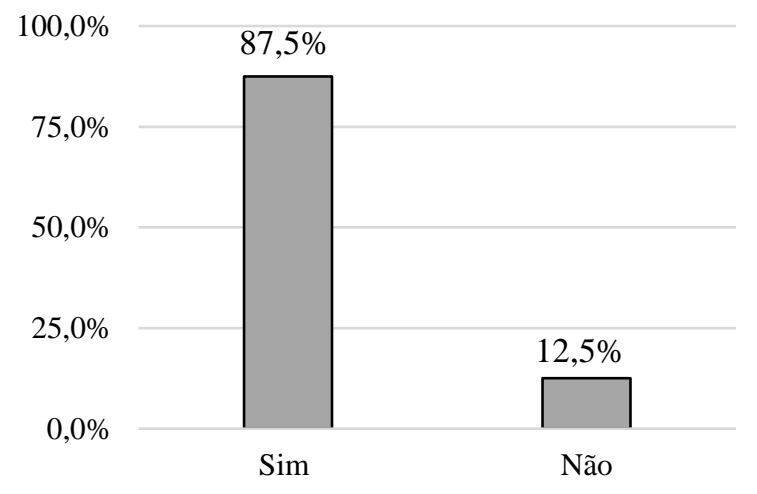

Figura 9: É aplicável no canteiro de obras.

\subsection{Pretensões de utilizar a Realidade Aumentada no canteiro de obras}

Após a realização do teste piloto e a experiência vivenciada in loco, a maioria manifestou pretensões em utilizar a RA na execução de obras futuras (Figura 10). Tal porcentagem pode ser justificada pelas vantagens apresentadas na utilização da RA na execução dos projetos; facilitando a leitura dos mesmos durante a execução, evitando assim os retrabalhos frequentes no dia a dia de obras.

Já os que optaram em não utilizar, ressaltaram as dificuldades apresentadas nos apps e que em breve, dependendo do desenvolvimento dos aplicativos, seu uso poderia ser viável. 


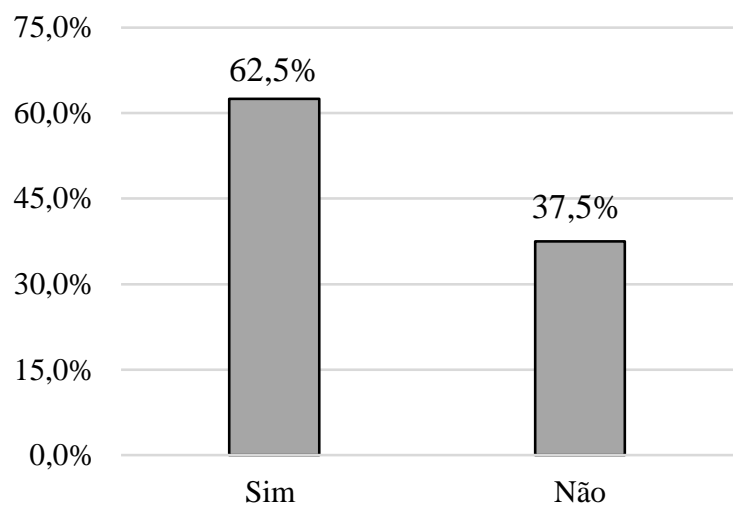

Figura 10: Tem pretensões de utilizar no canteiro de obras.

O estudo apontou que apesar de 50\% dos entrevistados possuir 36 anos ou mais, ou seja, profissionais experientes e atuantes no canteiro de obras, acostumados as práticas tradicionais da construção civil, mais de $85 \%$ foram favoráveis a aplicação da RA no canteiro, em razão dos benefícios que tal inovação pode proporcionar.

Importante destacar que embora mais de $40 \%$ da amostra seja composta por serventes, que geralmente representam os colaboradores com menor nível de qualificação dentro da obra, e que mais de $60 \%$ ainda não tinha utilizado a RA, mais de $60 \%$ indicou que tem pretensões de usar a RA no canteiro de obras.

\section{Conclusão e considerações finais}

O presente artigo tratou da aplicabilidade da RA com dispositivo móvel na fase de execução de obra que, apesar de algumas restrições no que diz respeito às propriedades das ferramentas, teve uma boa aceitação por parte dos trabalhadores entrevistados. Como resultado, observou-se um grande potencial de contribuição da RA no meio construtivo, associada a treinamentos que permitam aos usuários identificar detalhes necessários ou até falhas de projeto. No que tange as dificuldades apresentadas, constatou-se a necessidade de internet com boa capacidade e a dificuldade de posicionar os modelos. Diante do cenário apresentado, entende-se que esse processo se dará de forma lenta e gradual, principalmente em razão de que a utilização de novas tecnologias implica em investimentos para aquisição de equipamentos, treinamentos e licenças de softwares.

Isto posto, o estudo enfatiza a importância da inclusão da Realidade Aumentada no dia a dia do canteiro de obras, para um melhor entendimento dos projetos de arquitetura e engenharia, resultando assim na minimização de erros e retrabalhos.

Ao mesmo tempo que a RA e RV estão em evidência, surge a Realidade Mista, que une as duas primeiras e possibilita a inserção de objetos virtuais no mundo real. Assim, sugere-se como trabalhos futuros a utilização de outros apps voltados a realidade aumentada, virtual e mista, haja vista que sua junção potencializa mais ainda os ganhos com a utilização dessas tecnologias. 


\section{Referências}

[1] ASSIS, Jonas H. G. de; ANDRADE, Max L. V. de; BROCHARDT, Mikael M. de S. A. Aplicações de Realidade Aumentada no Canteiro de Obras: Proposta de utilização na visualização de projetos de instalações para a execução. SIGraDi 2016, XX Congress of the Iberoamerican Society of Digital Graphics, [s. 1.], p. 9-11, 2016.

[2] FREITAS, M. R. de; RUSCHEL, R. C. Validação de aplicativo comercial visando à incorporação da realidade aumentada a um modelo de avaliação pós-ocupação. Ambiente Construído, Porto Alegre, 2015. ISSN 1678-8621 Associação Nacional de Tecnologia do Ambiente Construído. http://dx.doi.org/10.1590/s1678-86212015000200016

[3] VIEIRA, H. F. Dinamização da Construção Civil Pela Tecnologia de Informação. In: Simpósio Brasileiro De Gestão E Economia Da Construção, 4.; Encontro LatinoAmericano De Gestão E Economia Da Construção, 1., Porto Alegre, 2005. Anais... Porto Alegre, 2005.

[4] WANG, X. et al. An Empirical Study on Designers' Perceptions of Augmented Reality within an Architectural Firm. Electronic Journal of Information Technology in Construction ITcon, v. 13 (special issue), December 2008. 536-552 pp. ISSN 1874-4753.

[5] MAYR, Luiz Roberto. Falhas De Projeto E Erros De Execução: Uma Questão de Comunicação. Orientador: Prof. Gregório Jean Varvakis Rados, Ph.D. 2000. Dissertação (Mestrado) - UFSC, Florianópolis, 2000.

[6] CUPERSCHMID, A. R. M.; FREITAS, M. R.; RUSCHEL, R. C. Tecnologias Que Suportam Realidade Aumentada Empregadas em Arquitetura e Construção. Caderno s PROARQ, v. 19, p. 47-69, 2012.

[7] CUPERSCHMID, A.R.M; FREITAS, M. R. de. Possibilidades de uso de Realidade Aumentada Móvel para AEC. In: Simpósio Brasileiro De Qualidade Do Projeto No Ambiente Construído, 3.; Encontro Brasileiro De Tecnologia De Informação E Comunicação Na Construção, 6., 2013, Campinas. Anais... Porto Alegre: ANTAC, 2013. p. 1-12.

[8] ALLEN, M.; REGENBRECHT, H.; ABBOTT, M. Smart-phone Augmented Reality for public participation in urban planning. In: 23RD Australian Computer-Human Interaction Conference. Proceedings... ACM, 2011.

[9] SHEN, Z.; JIANG, L. An augmented 3D iPad mobile application for communication, collaboration, and learning (CCL) of building MEP systems. Computing in Civil Engineering, 2012.

[10]RANKOHI, S.; WAUGH, L. Review and Analysis of Augmented Reality Literature For Construction Industry. Visualization in Engineering, springer 2013.

[11] YABUKI, N., HAMADA, Y., FUKADA, T.: Development of an accurate registration technique for outdoor augmented reality using point cloud data. In: Proceedings of the 14th International Conference on Computing in Civil and Building Engineering (2012)

[12] MICROSOFT (EUA). HoloLens 2: Uma nova realidade da computação. EUA: Microsoft, 2020. Disponível em: https://www.microsoft.com/pt-br/hololens. Acesso em: 27 jul. 2020.

[13] UNITY REFLECT (EUA). Unity Reflect: Crie experiências em 3D em tempo real, inclusive em AR e VR. EUA: Copyright (C) 2020 Unity Technologies, 2020. Disponível em: https://unity.com/pt/products/unity-reflect. Acesso em: 27 jul. 2020.

[14] GIL, Antônio Carlos. Como elaborar projetos de pesquisa. São Paulo: Atlas, 4a ed. 2002. $175 \mathrm{p}$. 